\title{
Allergien sind keine Bagatelle
}

$\mathrm{E}$ $s$ ist uns eine wahre Freude, Ihnen die aktuelle Ausgabe des Allergo Journal zur präsentieren. Sie belegt einmal mehr, welche Breite das Spektrum der allergischen Erkrankungen umfasst. Dass es sich hierbei oft um mehr als nur „ein bisschen“" Heuschnupfen und Augenjucken handelt, wird durch die unterschiedlichsten Überempfindlichkeitsreaktionen belegt, die einen lebensbedrohlichen oder tödlichem Verlauf nehmen. Zum Glück sind solche Verläufe relativ selten, dennoch ist jeder einzelne von ihnen einer zu viel. Eine der Gruppen, für die hier ein hohes Risiko besteht, sind Patienten mit schweren anaphylaktischen Reaktionen. Bei ihnen kann ein Mangel an Kenntnissen über Auslöser bzw. Allergene und über die richtige Selbtsmedikation fatal enden. Nationale und internationale Untersuchungen haben gezeigt, dass tatsächlich große Defizite in der Versorgung und Schulung dieser Patienten bestehen. Diese zu verbessern, hat sich die im Jahr 2008 gegründete Arbeitsgemeinschaft Anaphylaxie - Training and Education (AGATE) zum Ziel gesetzt. Mehr zum Hintergrund von AGATE, dem Anaphylaxie-Schulungsprogramm und den bisher erzielten Ergebnissen erfahren Sie im Interview mit Knut Brockow auf Seite 184.

Eine weitere Risikogruppe in Bezug auf einen potenziell letalen Ausgang der Allergie sind Patienten mit Arzneimittelreaktionen. Die Abklärung von Reaktionen, die im

„Dass es sich bei allergischen

Erkrankungen oft um mehr als nur ,ein bisschen' Heuschnupfen handelt, wird durch die Überempfindlichkeitsreaktionen belegt, die einen tödlichen Verlauf nehmen. "
Zusammenhang mit der Einnahme von Arzneimitteln auftreten, ist so sicher Teil unseres medizinischen Alltags, wie das Amen in der Kirche. In einer großen Mehrheit der Fälle handelt es sich um lästige, aber kontrollierbare Arzneimittelexantheme vom Spättyp, seltener um allergische bzw. pseudoallergische Soforttypreaktionen, die mit höherem Risiko einhergehen. Außerdem gibt es noch die zunehmend größer werdende Patientengruppe, bei denen fragliche Reaktionen nach Medikamenteneinnahme aufgetreten sind und die zur allergologischen Ausschlussdiagnostik vorgestellt werden. Hier kann man sich manchmal des Eindrucks nicht erwehren, dass die Diagnostik nicht nur den Patienten, sondern auch den Sicherheitsbedürfnissen der überweisenden Kollegen dient. Dass Arzneimittelreaktionen nicht auf die leichte Schulter genommen werden dürfen, zeigt der Beitrag von Maja

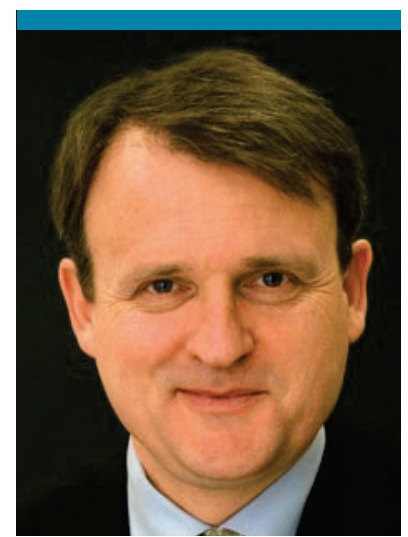

Prof. Dr. Thilo Jakob, Allergieabteilung \& Forschergruppe Allergologie, UniversitätsHautklinik Freiburg

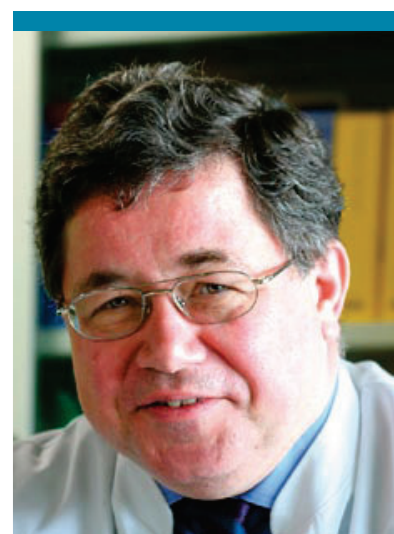

Prof. Dr. Hans F. Merk, Hautklinik der Medizinischen Fakultät, Universitätsklinikum der RWTH Aachen
Mockenhaupt und Yvonne Liß. Sie und ihre Teamkollegen vom Deutschen Dokumentationszentrum für schwere Hautreaktionen $(\mathrm{dZh})$ an der Universitäts-Hautklinik Freiburg erfassen alle schweren kutanen Arzneimittelreaktionen in Deutschland und verfügen über eine 20-jährige Erfahrung auf diesem schwierigen Gebiet. In dem ersten von zwei CME-Fortbildungsbeiträgen können Sie sich ab Seite 200 über die neusten Erkenntnisse zu den Themen Erythema exsudativum multiforme majus (EEMM), Stevens-Johnson-Syndrom (SJS) und Toxisch epidermale Nekrolyse (TEN) informieren. Ein zweiter Beitrag zur akuten generalisiserten exanthematischen Pustulose (AGEP) und und zur „drug reaction with eosinophilia and systemic reaction" (DRESS) wird bald folgen.

Schließlich erwarten Sie - hochaktuell und sehr spannend - die Abstracts zum 9. Arbeitsgespräch Insektengiftallergie am 20. und 21. Mai in Landshut (ab Seite 186). Hier hat es einen enormen Wissenszuwachs gegeben. Insbesondere die molekulare Charakterisierung der Giftkomponenten und die systematische Untersuchung von Risikofaktoren werden sich in einer verbesserten Diagnostik und einem besseren Patientenmanagement niederschlagen.

Viel Freude und Kenntnisgewinn bei der Lektüre wünschen

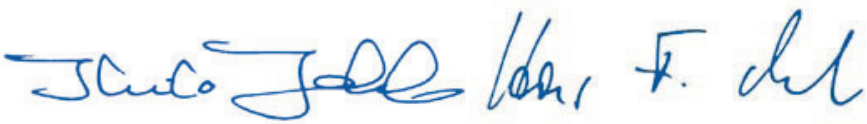

Prof. Dr. Thilo Jakob 